To appear in P.F. Dell, \& J. O'Neil (Eds.), Dissociation and the dissociative disorders: DSM-V and beyond.

CHAPTER 36

\title{
Opioid use disorder and dissociation
}

\author{
Eli Somer
}

This essay explores the inter-relationship between addiction to narcotics and dissociation. The focal premise is that drug use disorder, particularly opioid use disorder (OUD), is a condition driven by the need to chemically induce a state that mimics psychogenic dissociation. In other words, some individuals suffering from OUD are motivated to chemically induce a dissociative state to defend themselves against the memories of childhood abuse and the related pain experience. Consequently, I call this 'chemical dissociation'.

For the purposes of this chapter, dissociation is assumed to be a psychological defense mechanism developed in the face of extreme, or prolonged and inescapable, physical, sexual and emotional traumata. It works as a shield against the conscious experience of overwhelming stress by producing psychological and/or physical analgesia, emotional calming, and a breakdown of the normally integrated experiential components of behavior, affect, sensation, knowledge, and identity. The main thesis in this essay is that for many individuals suffering from OUD, chemical dissociation serves similar purposes by inducing powerful tranquilizing and numbing effects.

It is posited that in a certain subgroup of people, OUD constitutes the second stage of a strategy developed for coping with intolerable experiences and their memoriesWhen psychological dissociation is ineffective, or when substances that are able to produce rapid and efficient relief are available, some traumatized persons look beyond their own mental resources for relief. When these victims get access to consciousness-altering substances, they discover the immediate advantages of "chemical dissociation", and its rapid effect on both the body and the mind. This is when chemical dissociation can become a selfmedicating alternative. In such persons, the first stage psychogenic dissociation may manifest itself in the usual way, but it is more often masked by second stage chemical dissociation in the intoxicated state.

To substantiate this thesis I present data on common etiological roots of post-traumatic stress, dissociative disorders, and OUD; common neurobiological mechanisms of these disorders; their co-morbidity, and their similarities in the dimension of subjective experience (phenomenology).

\section{Common etiological roots}

People with a childhood trauma history constitute a significant proportion of opiod users (Van Hassselt, Ammerman, Glancy \& Bukstein, 1992). An overview of several epidemiological studies of comorbid posttraumatic stress disorder (PTSD) and substance use disorders (SUD) reveals that the odds of drug use disorders are three times greater in individuals with PTSD than in those without it (Chilcoat \& Menard, 2003). Gianconia et al. (2000), for example, reported that the data for co-morbid cases of SUD and PTSD in which PTSD was the primary disorder was $66.7 \%$. Four predominant hypotheses have been proposed to explain the consistent findings around PTSD-SUD comorbidity:

1. High-risk hypothesis - Drug use is a high-risk behavior that increases the risk for trauma exposure (Brown \& Wolfe, 1994)

2. Susceptibility hypothesis - Drug users are more susceptible to PTSD following exposure to threatening events (Brown \& Wolfe, 1994)

3. No direct relationship hypothesis - The association appears because both conditions derive from a third common factor (e.g., genetics)

4. Self-medication hypothesis - Individuals with PTSD use substances to control their emotional pain (Khantzian, 1985)

Using reports about the date of onset of the disorder, Kessler et al. (1995) estimated that among co-morbid PTSD-SUD subjects, PTSD occurred first in 53-65\% of the men, and in $65-84 \%$ of the women, arguing that PTSD generally predates SUD in those who experience both disorders. Based on their prospective study, Chilcoat \& Menard (2003) concluded that preexisting SUD does not significantly increase the risk for PTSD among adults relative to those without SUD. On the other hand, adults with a history of PTSD had a fourfold increase in the risk of SUD compared with those without PTSD. Hypotheses 1-3 do not predict such findings, while hypothesis 4 does, lending 
relative support for the self-medication hypothesis. Further evidence for a possible casual relationship between PTSD and SUD has been provided by Stewart, Conrod, Pihl, and Dongier (1999). They show that the severity of alcohol disorder symptoms correlates with the severity of PTSD arousal symptoms and that the severity of anxiolytic and analgesic dependence correlates with the severity of PTSD numbing and arousal symptoms. They also show how analgesic dependence severity correlates with PTSD intrusion symptoms. Other researchers (Liebschutz, et al, 2002; Malinkovsky-Rummel \& Hansen, 1993) have demonstrated that adult SUD is the most prominent consequence of childhood abuse.

The prominence of childhood trauma in the lives of recovering OUD patients has been demonstrated in three separate studies conducted in Israel. Data consistently showed higher traumatization history scores (typically reflecting childhood abuse and neglect) among patients recovering from OUD compared to those measured in consecutive admissions to outpatient stress clinics (Somer, 2004; Somer, Soref, \& Lawental, 2004; Somer \& Avni, 2003).

In sum, trauma history, particularly childhood trauma, would seem to be an etiological factor in both dissociative disorders and OUD. Significant statistical relationships exist among trauma history, severity of PTSD symptoms, and substance use disorder, especially the use of tranquilizing drugs.

\section{Trauma and the neurobiological basis for OUD}

How does trauma influence the biochemistry of the brain? Is there a possible connection between posttraumatic neuro-chemical changes in the brain and the proclivity to self-medicate with opiates? In the locus ceruleus, hippocampus, amygdala, and anterior hypothalamus, the areas of the brain most concerned with emotions and stress, there is a high density of both norepinephrine and opioid receptors (Langer, 1978). People with PTSD responding to cues associated with noxious stimuli trigger the firing of the locus ceruleus, releasing abnormal levels of key hormones involved in response to stress, such as norepinephrine (NE) (Cohen \& Servan-Schreiber, 1992). Van der Kolk, Greenberg, Boyd et al. (1985) suggested that physiological aspects of both opiate withdrawal and PTSD are related to central noradrenergic hyperactivity associated with a relative decrease in opioid receptor binding; such endogenous opioid release may result in a temporary sense of control. When people are in danger, they produce high levels of natural opioids, which can also temporarily mask physical pain. Scientists have found that people with PTSD continue to produce such higher levels even after the danger has passed; this may be associated with the blunted emotions associated with the condition (Yehuda, 1998). The relationship between peri- and post-traumatic secretion of natural opioids and dissociation has not yet been explored, but the role of dissociation in shielding individuals from the full impact of trauma has been discussed extensively in the literature (e.g., Chu \& Dill, 1990; Irwin, 1994; Herman, Perry, \& Van der Kolk, 1989; Putnam, 1995).

The animal model of inescapable shock parallels the equivalent of learned helplessness in humans and could shed further light on neurochemical mechanisms likely to be operating. Animals exposed to inescapable shock develop analgesia when exposed to another stressor shortly thereafter; this response is mediated by endogenous opioids and is reversed by naloxone (Van der Kolk \& Greenberg, 1987). Subsequent studies suggest that serious physical threats may induce analgesia and numbness in human victims as well (Cardeña et al., 1998; Cardeña \& Spiegel, 1993) and that anesthesia-analgesia were the symptoms that best predicted cases of post-traumatic dissociative disorders (Nijenhuis, Spinhoven, Vanderlinden, et al., 1998). In animal models of PTSD, numbness is likened to a conditioned analgesia in response to inescapable shock (Foa, Zinbarg, \& Rothbaum, 1992; Pitman, van der Kolk, Orr, \& Greenberg, 1990). Schiele (1992) even argued that chronic stress induces a physiological state that resembles dependency on high levels of exogenous opiates; and that when severely stressed, subsequent fear and the accompanying endogenous opioid secretion attenuates the perceived intensity of subsequent shocks.

All told, preliminary evidence exists to show that traumatized people produce elevated levels of natural opioids, which can temporarily mask emotional and physical pain. It is, therefore, conceivable that traumatized individuals would find the effect of exogenous opiates to be a gratifying shield between post-traumatic torment and conscious awareness

\section{Co-morbidity of dissociative disorders and OUD.}

If OUD and dissociative disoders have common etiological roots and if opioids (either endogenous or exogenous) are a natural recourse in the face of traumatic duress, it is only natural that OUD and dissociative disorders would co-occur. Most of the literature on dissociative disorders (DD), trauma, and addictions reveal a probabilistic concurrence of the 
phenomena. Ross, Kronson, Koensen, Barkman et al. (1992) reported that individuals who abuse alcohol and drugs have four times the incidence of child sexual abuse and three times the incidence of DD compared to non-clinical controls. In a survey of 185 individuals with severe dissociative disorders, $57 \%$ had problems with drug abuse (Rivera, 1991). Dunn, Paolo, Ryan, and Van Fleet (1995) showed that $41.5 \%$ of chemically dependent veterans received an average DES score of 15 or higher (DES range $0-100$ ), which is considered to be rather elevated. In comparison, Israeli Dissociative Disorders patients scored 29.45 on the DES (Somer, Dolgin, \& Saadon, 2001). The average total score on the Hebrew-Dissociative Experiences Scale (H-DES) for detoxified heroin users was 21.27 $(\mathrm{SD}=15.02)$ (Somer \& Avni, 2003). In comparison, Somer, Dolgin, \& Saadon (2001) reported mean HDES scores for two relevant groups as follows: Posttraumatic Stress Disorder and Acute Stress Disorder:20.36; Dissociative Disorders: 29.45.

A newly developed tool for the assessment of pathological dissociation, the Hebrew version of the Multidemensional Inventory of Dissociation (H-MID, Somer \& Dell, in press) has a range of $0-100$, with scores of 21 or higher indicative of possible dissociative psychopathology. A study was conducted in Israel comparing a recovering heroin users group,to respondents sampled from stress and trauma clinics. One in three of the ex-heroin users scored 21 or higher on the H-MID, compared to only one in ten for the stress and trauma clinic controls (Somer, Soref, \& Lawental, 2004). The same study similarly indicated that ex-heroin users showed significantly higher scores on post-traumatic measures of avoidance, intrusion and hyper-arousal than the stress and trauma clinic controls. The same ex-heroin users sample was also statistically more disturbed than stress and trauma clinic controls on the following H-MID clinical scales: memory problems, flashbacks, somatoform dissociation, identity confusion, ego-alien experiences, selfalteration, self-states and alters, and Schneiderian firstrank symptoms.

To recapitulate, a history of childhood trauma and dissociative psychopathology seem to be risk factors for OUD. The insufficiency of psychogenic dissociation in the protection of the conscious mind could be a pathway to OUD. Deficiencies in dissociative defenses may be the consequence of great post-traumatic suffering that cannot be effectively contained, or of meager dissociative capacities. Thus, we hypothesize that some survivors of child abuse self-medicate with opiates to mimic chemicals endogenously released to attenuate traumatic distress. This hypothesis predicts that individuals with OUD would actually seek out and go through dissociative experiences during opiate use, a testable prediction.

\section{Similar phenomenology.}

The similarity between chemically induced and psychologically induced symptoms of dissociation required special underscoring of the disclaimers to the instructions on scales measuring dissociation, which ask respondents to endorse items only if the described experiences did NOT occur under the influence of alcohol or drugs (e.g., DES; Carlson \& Putnam, 1993, MID; Dell, 2004; H-MID; Somer \& Dell, in press).

To study the meaning and function of opioid use among a group of Israelis recovering from a heroin use disorder, we conducted five focus groups comprised of 10-15 individuals each. In total, we met 66 consenting persons receiving outpatient treatment in private and government drug rehabilitation clinics ${ }^{1}$. These individuals had abused alcohol, cannabis, amphetamines, and opiates, but for 60 (91\%) participants, heroin was the preferred drug. Fifty-nine participants $(89 \%)$ had a history of child abuse. I asked them to describe their experience with the drugs. Specifically, I asked them to tell me what effects and sensations of the drugs had been most meaningful or important to them. In other words, what functions had the drugs fulfilled? — what role had they fulfilled? Their discussions were audio-recorded and transcribed verbatim. Analysis of the text was conducted in two main steps. In the first phase, I read the transcribed discussions of the five groups and systematically coded and sorted the material into key themes by means of cross-case analyses with a constantcomparison method. Core themes were identified and compared, and analytical categories were later illustrated / exemplified by specific quotations (Krueger, 1994).

Four main themes emerged from the focus groups in regard to their experience with drugs: (1) chemical amnesia, (2) chemical suppression of post-traumatic arousal symptoms, (3) chemical numbing, depersonalization and derealization, and (4) soothing, gratifying pleasure. The themes will be presented with illustrative, representative quotes.

\footnotetext{
${ }^{1}$ The author wishes to thank Ronit Avni, MSW, for contributing the idea for this study.
} 


\section{(1) Chemical amnesia.}

One recovering heroin user described how the substance has helped him not to remember current and past feelings and thoughts:

Heroin helps me forget the pain in my life. Soon after the injection I feel I can stop thinking about the troubles ... the horrible memories ... without heroin I couldn't leave home. I was simply too overwhelmed.

He had experienced the drug as a means of distancing himself from incapacitating memories and of reducing conscious awareness of current troubling issues.

Here is how another recovering user described the amnestic function of her drug habit:

When I am high I don't care about the horrors of my childhood. I manage to ignore my fears as if they had never been there, I can't remember my nightmares ... geez, I hate those nightmares ... they keep haunting me for years. Only when I'm on heroin I get not to think about them ... It is as if I never dreamt them in the first place ... and my daily problems with bills and my family become so small I could hardly care about them. They just fade away.

This individual uses the substance to induce a memory disorder that shields her against chronic nightmares concerning childhood traumata. It seems that she also finds the amnestic agent useful in helping her forget about current life problems and challenges. A habit that was conceivably developed to deal with posttraumatic distress has evolved into an apparently maladaptive means to cope with daily stressors.

\section{(2) Chemical suppression of post-traumatic arousal symptoms.}

Several respondents described heroin as their preferred calming agent and as an effective course towards controlling a variety of post-traumatic symptoms, representing both sympathetic and affective arousal. Here is what one 35-year-old participant, a survivor of child abuse, and a convicted wife beater said when he talked about the role that heroin played in the management of his explosive anger:

I have always been a very angry person. Often I can't control my temper. I feel that the anger consumes me ... . I feel like I want to get even. It's like I constantly want to beat the $\mathrm{s}^{* * *}$ out of someone. Anyone. I have so much hate in me, it consumes me. After I shoot up, the anger doesn't bother me any more. It's like I disconnect it. I turn it off. Maybe it is still there, but I am elsewhere, unbothered by it.

This man describes life-long problems with violent anger. He portrays heroin use as a process of selfmedication. The burning and distressful drive for violence ("it consumes me") only subsides following the use of narcotics. This man eloquently portrays a dissociative experience when he acknowledges the existence of the dissociated anger during the consciousness-altered state.

A 28-year-old woman, an abandoned child that lived her entire childhood in foster homes, portrayed her heroin intoxication in terms of its role in controlling fears, anxieties, and an overall tense condition:

I suffer from many fears. Men scare me, I can't trust women, and children make me feel uneasy, I hate the night, I can't stand crowds and I dread doctors and dentists. I am constantly on edge. On guard. As soon as I get high I, umm ... nothing bothers me anymore. I can calm down and it's like I am able to deal with many of those fears much better ... sometimes I also get a feeling that nothing scares me anymore. It's such a relief to be able to unwind from all my stresses and get a real break from my tensions ... it sure beats sleep.

This woman describes many fears triggered by others. In a world crowded by human beings her fears seem to be constantly triggered. Simple interactions, even with ostensibly innocuous figures (e.g., children) produce distress to a point where she cannot receive proper medical care without the experience of considerable discomfort. Her only gratifying respite from her steady state of hyper-vigilance and hyper-arousal is opiate intoxication.

\section{(3) Chemical numbing, depersonalization, and derealization.}

Most comments on the experience sought in or felt during heroin use were also characteristic of classical dissociative experiences. One 50-year-old recovering user, an incest survivor, described how a variety of somatic problems had been temporarily eased by the drug use:

I always walk around with a splitting headache. No pain medication has ever helped me. I also have this other problem, it is as if I have extensive burn wounds, my skin is sore, I can't be touched ... . I think I might be arthritic, too. I have been roughed up pretty badly throughout my life. I think that my joints are a total mess ... . Heroin 
lets me not feel these pains and aches. For a few hours I am fairly well. I can rest.

I hate my sexuality, my sex drive makes me sick ... . When I'm high I am not in touch with my real sexual drive. I prefer it that way.

Heroin is described here as a preferred analgesic and as an effective depressing agent for her conflicted sexual drive. Several participants talked about depersonalizing and derealizing effects that they derived from heroin use. Here is what a 37-year-old former prostitute had to say:

I can disregard my emotions when I am on the drug. I couldn't care less about myself because I hardly felt myself. It is pretty awful to be myself you know ... . I'd rather not feel the stuff that makes me feel so bad, the sadness and the emptiness, those endless internal quarrels. This white stuff helps me not to feel my depression, I can ignore all the threats and the dangers in this

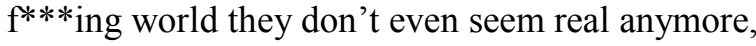
I couldn't give a $\mathrm{s}^{* * *}$ about nothing.

This world is full of all sorts of creeps who always want to $\mathrm{f}^{* * *}$ you or hurt you. When I was on the drug, they could take my body and do what they

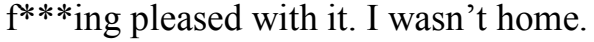

This individual convincingly tells the interviewer how she yearned to distance herself from her emotional pain by the disconnecting effect of the opiate. Ironically, this survivor of child abuse is being continuously revictimized in her sex trade profession. Chemical depersonalization helps her tolerate the unsavory experiences involved in providing sexual services to her customers.

\section{(4) Soothing, gratifying pleasure.}

Heroin did not only help our respondents in numbing their pain and in distancing them from their painful memories, but also helped generate pleasurable feelings that were ordinarily so scarce in their lives. Here is what a 40-year-old man, who grew up in an extremely neglectful and emotionally abusive family, said:

I always feel cold. I am cold from the inside ... I feel so empty.

When I shoot up, I have this great rush of warmth building up at the pit of my stomach. It radiates inside as if I have an internal fireplace. It's so comforting.
His description of the appealing warmth of the drug can only be appreciated when contrasted with the portrayed coldness inside him. This same man also talked about how he had yearned to be hugged and comforted by his mother whom he also depicted as a "cold bitch."

It seemed that some participants had anthropomorphized the heroin and had perceived it as having benevolent traits. One recovering heroin user shared the following observations about his unresolved craving for the substance:

Nothing beats the feeling I got from heroin ... just found this good stuff irresistible ... like a gentle blanket that was lovingly wrapped around my shoulders it covered my entire body, it calmed me down, sometimes it glowed from my within.

There was something sweet about the seduction of the stuff. I am not sure I can describe it to you but imagine being caressed from inside.

This man described the substance as a "loving" protective entity that is capable of radiating soothing warmth. His account of the opiate also portrays a "being" that is quite sensual, almost erotic.

The results of this qualitative inquiry render credible preliminary support to the idea that some survivors of childhood abuse or neglect utilize heroin defensively as a dissociating agent, specifically seeking the numbing, depersonalizing, and amnestic capacities of the drug. Moreover, a few survivors that were recovering from their addiction assigned benevolent nurturing human qualities to the drug. This was a sad testimonial to the emotional emaciation of these individuals.

Preliminary controlled research provided some quantitative support for these qualitative findings. Somer and Avni (2003) demonstrated that respondents with higher trauma scores were more likely to report dissociative experiences during their drug use (chemical dissociation). That is to say, traumatized users tended to experience heroin as producing more dissociative experiences than users with a milder trauma history. Specifically, these drug-free high dissociators were more likely to report, retrospectively, depersonalizing and derealizing experiences when they had been using drugs. Furthermore, stronger dissociative experiences during drug use were found to be related to greater psychological distress during inter-use craving (non withdrawal-related). This datum demonstrates the possible shielding function of the narcotic for users who are survivors of child abuse. For 
respondents who tended to experience higher levels of chemical dissociation during heroin use, being off the drug was more distressful than for users who had experienced lower levels of chemically induced dissociation. Interestingly, a statistical relationship was also discovered between retrospectively reported psychological distress, experienced during inter-use craving, and current psychological dissociation, which was measured in a detoxified state. This may imply that when these individuals attempt to relinquish their chemical dissociation by detoxification, they may experience an increase in distress that is predictive of their likelihood to employ psychological dissociation later on during their recovery. That is, the two dissociative mechanisms, exogenous and endogenous, are more likely to be measured in survivors of childhood trauma and they are interchangeable. If specific treatment for post-traumatic dissociation is not provided, detoxifying survivors may tend to substitute one type of dissociative defense with another.

The employment of psychological dissociation by survivors of child abuse who are recuperating from OUD may compromise the stability of their recovery. Dissociation has been found to be an independent predictor of abstinence from heroin use (Somer, 2003). The two variables appear to be inversely related: recovering heroin-dependent persons who were high dissociators are less likely to enjoy favorable treatment outcomes as reflected in extended periods of abstinence. Dissociative psychopathology may be conducive to relapse by compromising the awareness of rehabilitating opiate users to high-risk situations. It may also impair the quality of control over their behavior. Alternatively, and as posited earlier, persons who had only recently become abstinent could be more likely to compensate for the missing chemical dissociation by evoking their psychogenic traumarelated dissociative defenses.

Further support to the interchangeability of psychogenic and chemical dissociation in the lives of recovering opiate users was provided by Somer, Soref $\&$ Lawental (2004). The investigators compared dissociative psychopathology across three groups of recovering heroin users: (1) Individuals on an agonist treatment; these respondents received Methadone, a synthetic, less addictive form of heroin, to keep them from uncontrolled use of street drugs; (2) Individuals in early full remission who were treated in a controlled environment (in-patient detoxification and rehabilitation) and; (3) People in early full remission who were drug-free for more than 6 months. The first two groups had enjoyed some form of support and containment, either through the ingestion of heroinmimicking substances prescribed by the clinics that were treating them or through the professional boundaries and support that were provided in the total environment of the detoxifying agency. The third group had to rely more profoundly on their own internally-developed coping mechanisms. Compared to the first two research groups, these respondents reported the highest levels of psychoticism, hostility, anxiety (both general and phobic), post-traumatic intrusions, avoidance, and hyperarousal. They also scored highest on the following clinical scales of the H-MID: Memory problems, flashbacks, somatoform dissociation, identity confusion, ego-alien experiences, self-alteration, self-states and alters, and Schneiderian first-rank symptoms, revealing significant dissociative psychopathology. Given that the three groups did not differ significantly on biographic indices, length of treatment, severity of drug use disorder, or trauma history, I conclude that the elevated general, posttraumatic and dissociative psychopathology was best accounted for by the want of critical sustaining factors: the strong boundaries of a treatment milieu or (synthetic) chemical dissociation. This dearth of external coping resources probably played a significant part in the exacerbation of their pre-existing dissociative and related psychopathology.

The implications of the material presented in this chapter are important for the design of effective clinical practice with OUD patients. It is sensible to screen candidates for substance abuse treatment for post-traumatic and dissociative pathology. Before clinicians encourage recovering traumatized patients with drug use disorders to give up their chemical dissociation, they should vigorously address their underlying post-traumatic problems. Clearly, without a thorough resolution of trauma-related dissociation, a timely solution for the underlying psychopathology and the successful treatment outcome are less likely.

\section{References}

Cardenña, E., Holen, A., McFarlane, A., Solomon, Z., Wilkinson, C., \& Spiegel, D. (1998). A multisite study of acute stress reaction to a disaster. In Sourcebook for the DSM-IV, Vol. IV. Washington, DC: American Psychiatric Association.

Cardenña, E., \& Spiegel, D. (1993). Dissociative reactions to the San Francisco Bay area earthquake of 1989. American Journal of Psychiatry, 150, 474-478. 
Carlson, E. B. \& Putnam, F. W. (1993). An update on the Dissociative Experiences Scale. Dissociation, $5,116-127$.

Chilcoat, H. D. \& Menard, C. (2003). Epidemiological investigations: Co-morbidity of post-traumatic stress disorder and substance use disorder. In P. Ouinmette and P. J. Brown (Eds.) Trauma and substance abuse: Causes, consequesnces, and treatment of co-morbid disorders. Washington: American Psychological Association, pp 9-28.

Cohen J.D., \& Servan-Schreiber, D, (1992). Aneural network model of disturbances in the processing of context in schizophrenia. Psychiatric Annals, 22, 131-136,

Chu, J. A., \& Dill, D. L. (1990). Dissociative symptoms in relation to childhood physical and sexual abuse. American Journal of Psychiatry, 147, 887-892.

Dell, P. F. (2004b?) Multidimensional Inventory of Dissociation (MID): A comprehensive measure of the subjective/phenomenological domain of dissociation. Submitted for publication.

Dunn, G. E., Paolo, A. M., Ryann, J. J. \& Van Fleet, J. (1995). Co-morbidity of dissociative disorders among patients with substance use disorders. Psychiatric Services, 46(2), 153-156.

Foa, E. B., Zinbarg, R., \& Rothbaum, B. (1992). Uncontrollability and unpredictability in posttraumatic stress disorder: An animal model. Psychological Bulletin, 112, 218-238.

Gianconia, R. M., Reinherz, H. Z., Hauf, A. C., Paradis, A. D., Wasserman, M. S., \& Langhammer, D. M. (2000). Co-morbidity of substance use and post-traumatic stress disorders in a community sample of adolescents. American Journal of Orthopsychiatry, 70, 253-262.

Herman, J. L., Perry, J. C., \& Van der Kolk, B. A. (1989). Childhood trauma in borderline personality disorder. American Journal of Psychiatry, 146, 490-495.

Irwin, H. J. (1994). Proneness to dissociation and traumatic childhood events. Journal of Nervous and Mental Disease, 182, 456-460.

Krueger, R. A., (1994). Focus groups: A practical guide for applied research $\left(2^{\text {nd }}\right.$ ed.). Thousand Oaks, CA: Sage.

Langer, S.Z. (1978). Morphine and beta endorphin inhibit release of noradrenaline from cerebral cortex but not of dopamine from rat striatum. Nature, 271, 559-560.

Liebschutz, J., Savetsky, J. B., Saitz, R., Horton, N. J., Lloyd-Travaglini, C. \& Samet, J. H. (2002). The relationship between sexual and physical abuse and substance abuse consequences. Journal of Substance Abuse Treatment, 22, 121-12.

Malinovsky-Rummel, R. \& Hansen, D. J. (1993). Long-term consequences of childhood physical abuse. Psychological Bulletin, 14(10), 68-79.

Nijenhuis, E. R. S., Spinhoven, P., Vanderlinden, J., Van Dyck, R., \& Van der Hart, O. (1998a). Somatoform dissociative symptoms as related to animal defensive reactions to predatory threat and injury. Journal of Abnormal Psychology, 107, 6373.

Pitman, R. K., Orr, S. P., van der Kolk, B. A., Greenberg, M. S., Meyerhof, J. L., \& Mougey, E. H. (1990). Analgesia: A new dependent variable for the biological study of post-traumatic stress disorder. In M. E. Wolf, \& E. D. Mosnaim, (Eds), Post-traumatic stress disorder: Etiology, phenomenology, and treatment (pp. 141-147). Washington: American Psychiatric Publishing.

Putnam, F. W. (1995). Development of dissociative disorders. In D. Ciccheti \& D. J. Cohen (Eds.). Development psychopathology, Vol. 2. Risk, disorder, and adaptation. (pp. 581-608). New York: Wiley.

Rivera, M. (1991). Multiple personality disorder and the social systems: 185 cases. Dissociation, 4(2), 79-82.

Ross, C. R., Kronson, J., Koensen, S., Barkman, K., Clark, P. \& Rockman, G. (1992). Dissociative comorbidity in 100 chemically dependent patients. Hospital and Community Psychiatry, 43(8), 840842.

Schiele, D. R. (1992). The neuropsychobiology of addication, trauma and dissociation. Paper presented at the 5th Annual Western Clinical Conference on Multiple Personality and Dissociation, Costa Mesa, CA, April 10-12.

Somer, E. (2003). Prediction of abstinence from heroin addiction by childhood trauma, dissociation, and extent of psychosocial treatment. Addiction Research and Theory, 11(5), 339-348.

Somer, E. \& Avni, R. (2003). Dissociative phenomena among recovering heroin users and their 
Ch. 36, Somer E, Opioid use disorder and dissociation 8

relationship to duration of abstinence. Journal of Social Work Practice in the Addictions, 3(1), 2538.

Somer, E. \& Dell (In press). Development of the Hebrew-Multidimensional Inventory of Dissociation (H-MID): A valid and reliable measure of pathological dissociation. Journal of Trauma and Dissociation.

Somer, E., Dolgin, M., \& Saadon, M (2001). Validation of the Hebrew version of the Dissociation Experiences Scale (H-DES) in Israel. Journal of Trauma and Dissociation, 2(2), 53-66.

Somer, E., Soref, E., \& Lowental, E. (2004).

Dissociative disorders among individuals with opiate use disorder: A research report. Jerusalem: The Israel Anti-drug Authority (In Hebrew).

Stewart, S. H., Conrod, P. J., Pihl, R. O., \& Dongier, M. (1999). Relationship between post-traumatic stress symptom dimensions and substance dependence in a community-recruited sample of substance-abusing women. Psychology of Addictive Behaviors, 13, 78-88.

Van der Kolk, B.A. \& Greenberg, M.S. (1987). Psychobiology of the trauma response: Hyperarousal, constriction and addiction to trauma, traumatic reexposure. In B.A. Van der Kolk (Ed.), Psychological trauma. Washington, D.C.: American Psychiatric Press, pp 63--87.

Van der Kolk B.A., Greenberg M.S., Boyd H., et al. (1985). Inescapable shock, neurotransmitters and addiction to trauma: Towards a psychobiology of post traumatic stress. Biological Psychiatry 20, 314-325.

Van Hasselt, V B., Ammerman, R. T., Glancy, L. G. \& Bukstein, O. G. (1992). Maltreatment in psychiatrically hospitalized dually diagnosed adolescent substance abusers. Journal of the American Academy of Child and Adolescent Psychiatry, 31(5), 868-874.

Yehuda R. (1998). Psychoneuroendocrinology of post-traumatic stress disorder. Psychiatric Clinics of North America, 21(2), 359-79.

Author's note: Part of the data presented in this essay are a result of a study supported by the Israel AntiDrug Authority 